\title{
Activation of neutrophils by soluble and insoluble immunoglobulin aggregates from synovial fluid of patients with rheumatoid arthritis
}

\author{
John J Robinson, Fiona Watson, Mark Phelan, Roger C Bucknall, Steven W Edwards
}

\begin{abstract}
Objectives-Previous work has shown that synovial fluid isolated from patients with active rheumatoid arthritis contains soluble (not sedimented by centrifugation at $11600 \mathrm{~g}$ for two minutes) and insoluble (sedimented by centrifugation at $11600 \mathrm{~g}$ for two minutes) immunoglobulin aggregates that are capable of activating reactive oxidant production by bloodstream neutrophils. The purpose of this study was to determine which of these types of immunoglobulin aggregates activated the secretion of reactive oxygen metabolites and granule enzymes from neutrophils.
\end{abstract}

Methods-Cell free synovial fluid (from patients with rheumatoid arthritis) was added to neutrophils isolated from blood of healthy controls that had been incubated in the presence and absence of granulocyte-macrophage colony stimulating factor (GM-CSF). Reactive oxidant production was measured by luminol chemiluminescence (which detects both intracellular and extracellular oxidant production) and by cytochrome $c$ reduction (which measures superoxide secretion).

Results-The soluble aggregates only activated neutrophils that were previously primed, and activated a rapid and transient burst of reactive oxidant secretion. On the other hand, the insoluble aggregates activated primed and unprimed neutrophils with similar efficacy and most of the oxidants generated (especially in unprimed cells) were intracellular. The soluble aggregates, but not the insoluble aggregates, also activated the secretion of myeloperoxidase from neutrophils that had either been pretreated with cytochalasin $B$ or primed with GM-CSF.

Conclusion-It is thus proposed that these soluble immunoglobulin aggregates are responsible for activation of the release of tissue damaging granule enzymes and reactive oxidants from primed neutrophils within the rheumatoid joint.

(Ann Rheum Dis 1993; 52: 347-353)

As well as their important role in host defence during the killing of microbial pathogens, inappropriate infiltration and activation of neutrophils in tissues has been implicated in the pathology of inflammatory diseases such as rheumatoid arthritis. ${ }^{12}$ Thus it is envisaged that during active phases of the disease, these cells enter the joint and become activated to secrete their granule enzymes and reactive oxygen metabolites which then attack joint tissues. Indeed, many lines of evidence substantiate this model of tissue damage mediated by neutrophils-for example, neutrophils isolated from the synovial fluid of patients with rheumatoid arthritis exhibit biochemical properties indicating that they have been primed and activated in vivo ${ }^{3-6}$; synovial fluid contains oxidatively damaged components $^{78}$; and neutrophil derived enzymes such as myeloperoxidase are detectable within these joints ${ }^{910}$ in a molecular form indicating that degranulation has occurred in parallel with the secretion of reactive oxidants.

If neutrophils within synovial fluid have in fact been activated to secrete these toxic compounds, then there must be neutrophil activating factors present within such joints to account for this activation. Many independent lines of research have implicated large immune complexes, which are present in these joints in large quantities, as the factors that elicit these neutrophil responses. ${ }^{10-14}$ Although these insoluble complexes (those sedimented by centrifugation at $11600 \mathrm{~g}$ for two minutes) are indeed capable of activating neutrophils in vitro, they are very poor activators of secretion $^{14}$ of either reactive oxidants or granule enzymes: most of the reactive oxidant production is intracellular. Hence, such intracellular generation of reactive oxidants that is stimulated by these complexes is unlikely to be of importance in disease pathology.

We have recently shown that synovial fluid isolated from patients with rheumatoid arthritis contains various immunoglobulin aggregates that are capable of activating reactive oxidant generation in neutrophils. ${ }^{15}$ Some of these are sedimented by centrifugation at $11600 \mathrm{~g}$ for two minutes (here defined as insoluble) whereas others are not sedimented under these conditions (here defined as soluble). Curiously, the soluble aggregates only activate neutrophils that have previously been primed in vitro by agents such as granulocyte-macrophage colony stimulating factor or $\gamma$-interferon, or in vivo by cytokines, which are known to be present within these joints. The mechanisms of activation of neutrophils by these soluble and insoluble 
complexes seem to be biochemically distinct because (a) the kinetics of activation are different (the soluble complexes activate a rapid burst of oxidant production, whereas the insoluble complexes activate the cells more slowly); (b) the soluble complexes only activate previously primed cells whereas the insoluble complexes activate primed or unprimed cells with similar efficacy; (c) activation via the soluble complexes is staurosporine insensitive (and hence independent of protein kinase C) whereas that occurring via the insoluble complexes is staurosporine sensitive. In view of these apparently different activation mechanisms, the aim of this work was to determine if these soluble complexes activated the secretion of reactive oxidants and granule enzymes from neutrophils.

\section{Materials and methods}

PATIENTS

The group studied (30 patients) were those attending the rheumatology clinics at the Royal Liverpool Hospital. Rheumatoid arthritis was diagnosed from the American Rheumatism Association criteria. The mean age was 55 (21-80) years and the mean duration of disease was $7 \cdot 6(0.5-20)$ years. All had classical or seropositive rheumatoid arthritis. Control blood was taken from laboratory personnel.

\section{PREPARATION OF NEUTROPHILS}

Neutrophils were isolated from heparinised venous blood of healthy volunteers with Mono Poly-Resolving Medium ${ }^{16}$ exactly as described in the manufacturer's instructions. The process involved the separation of neutrophils via density gradient centrifugation at $600 \mathrm{~g}$ for 25 minutes. The neutrophil band was removed and subjected to hypotonic lysis to eliminate contaminating erythrocytes. After purification neutrophils were washed and resuspended in RPMI 1640 medium (containing $20 \mathrm{mM}$ HEPES) and their purity $(>97 \%)$ and viability $(>95 \%)$ assessed by May-Grünwald/Giemsa staining and trypan blue exclusion respectively. Cells were counted with a Fuchs-Rosenthal haemocytometer slide, and used within five hours of preparation.

\section{PREPARATION OF SYNOVIAL FLUID}

Synovial fluid was collected into heparinised tubes by aspiration of joints from patients with knee effusions. The fluid was then centrifuged at $600 \mathrm{~g}$ for 10 minutes to pellet the cells. The cell free fluid (immediately or after storage in aliquots at $-20^{\circ} \mathrm{C}$ ) was used intact in neutrophil activation studies or fractionated by centrifugation at $11600 \mathrm{~g}$ for two minutes. The resultant supernatant was retained and the pellet was resuspended to an equivalent volume in RPMI 1640 medium.

\section{NEUTROPHIL PRIMING}

Suspensions of neutrophils were incubated for one hour at $37^{\circ} \mathrm{C}$ in RPMI 1640 medium at $10^{7}$ cells $/ \mathrm{ml}$. Control suspensions contained no further additions and primed neutrophils were supplemented with $50 \mathrm{U} / \mathrm{ml}$ recombinant (r) GM-CSF. ${ }^{17}$ After incubation under these conditions the ability of the cells to generate reactive oxidants was assessed.

\section{MEASUREMENT OF REACTIVE OXIDANT} GENERATION

Chemiluminescence was performed on suspensions of neutrophils $\left(5 \times 10^{5} / \mathrm{ml}\right)$ suspended in RPMI 1640 medium containing $10 \mu \mathrm{M}$ luminol ${ }^{18}$ in a total volume of $1 \mathrm{ml}$ at $37^{\circ} \mathrm{C}$. After the addition of stimuli, photon emission was measured at $37^{\circ} \mathrm{C}$ with a 25 channel LKB Wallac 1251 luminometer. Superoxide production was measured as the rate of superoxide dismutase inhibitable cytochrome $c$ reduction. ${ }^{19}$ Suspensions $(1 \mathrm{ml})$ in both sample and reference cuvettes contained $75 \mu \mathrm{M}$ cytochrome $c$ and $5 \times 10^{5}$ cells in RPMI 1640 medium, and at time zero the stimulus was added. Reference cuvettes also contained $30 \mu \mathrm{g} / \mathrm{ml}$ superoxide dismutase (SOD), and the increase in absorption at $550 \mathrm{~nm}$ was measured with a Perkin-Elmer Lambda 5 spectrophotometer. In some experiments neutrophils $\left(1-5 \times 10^{5}\right)$ in RPMI containing 75 $\mu \mathrm{M}$ cytochrome $c$ and stimuli were suspended in a total volume of $200 \mu \mathrm{l}$ and increases in absorption at $550 \mathrm{~nm}$ (reference $450 \mathrm{~nm}$ ) were measured in a Bio-Rad 3550 kinetic plate reader. ${ }^{14}$ Appropriate control wells also contained $30 \mu \mathrm{g} / \mathrm{ml}$ SOD and absorption values in these wells were subtracted to calculate SOD inhibitable superoxide production.

MEASUREMENT OF MYELOPEROXIDASE RELEASE Degranulation of myeloperoxidase was measured as described by Edwards $e t a l^{20}$ after stimulation of neutrophil suspensions by fMetLeu-Phe $(1 \mu M)$ or immunoglobulin aggregates from rheumatoid synovial fluid in the presence and absence of cytochalasin B (5 $\mu \mathrm{g} / \mathrm{ml}$ ). Myeloperoxidase activity in the cell free supernatants was assayed by the guaiacol method. ${ }^{21}$

\section{Results}

ACTIVATION OF CHEMILUMINESCENCE AND

$\mathrm{O}_{2}{ }^{-}$SECRETION BY SYNOVIAL FLUID

When (unprimed) bloodstream neutrophils were incubated with cell free synovial fluid from patients with rheumatoid arthritis, luminol chemiluminescence was slowly activated, reaching a maximal value 15-20 minutes after addition (fig 1A). In parallel experiments, we showed that such synovial fluids failed to stimulate the secretion of $\mathrm{O}_{2}{ }^{-}$ (fig 1B). Because luminol meaures both intracellular and extracellular reactive oxidant production, ${ }^{18} 22$ the chemiluminescence shown in fig $1 \mathrm{~A}$ (open circles) is due to intracellular oxidant production because this is not accompanied by increases in cytochrome $c$ reduction, which is specific for the secretion of 

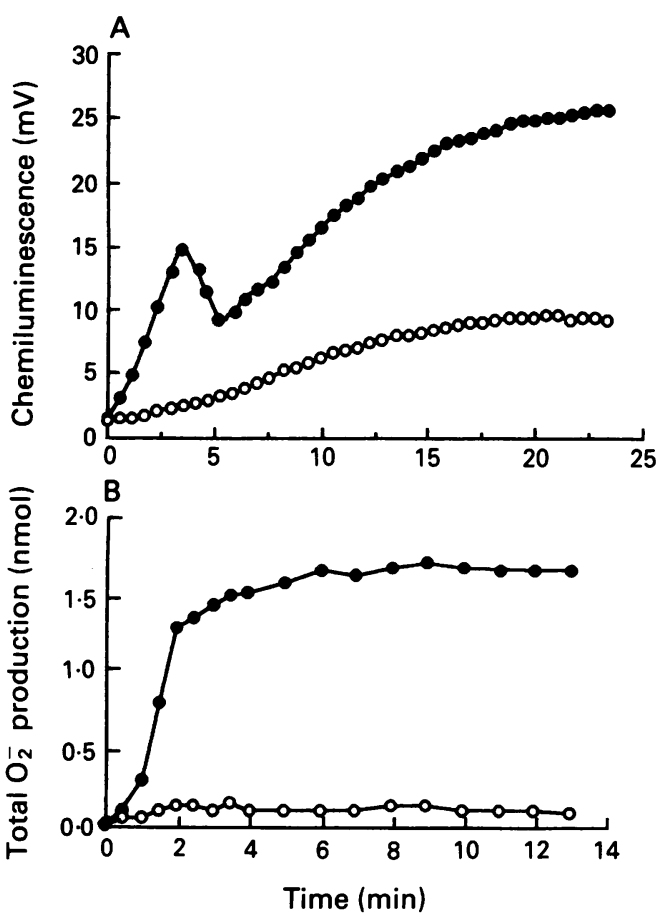

Figure 1 Activation of neutrophils by rheumatoid synovial fluid. Neutrophils $\left(10^{7} / \mathrm{ml}\right)$ were isolated from the blood of a healthy volunteer and suspended in RPMI 1640 medium in the absence $(0)$ or presence $(\bullet)$ of $50 \mathrm{U} / \mathrm{ml}$ granulocytemacrophage colony stimulating factor (GM-CSF). After incubation for one hour at $37^{\circ} \mathrm{C}, 5 \times 10^{5}$ cells were activated with $10 \%(v / v)$ cell free synovial fluid and the oxidants produced measured by $(A)$ chemiluminescence (LKB 1251 luminometer) and (B) cytochrome c reduction at $550 \mathrm{~mm}$ (Bio-Rad 3550 kinetic plate reader). In (A) $10 \mu M$ luminol was present and the volume was made up to $1 \mathrm{ml}$ with RPMI 1640 medium. In (B) $75 \mu M$ cytochrome c was present and the volume was made up to $200 \mu \mathrm{l}$ with RPMI 1640 medium: reference wells contained the same additions plus $30 \mu \mathrm{g} / \mathrm{ml}$ superoxide dismutase. Both assays were performed at $37^{\circ} \mathrm{C}$. Similar results were obtained in at least 20 further experiments with different synovial fluid samples and neutrophils from different donors.

$\mathrm{O}_{2}^{-}$When neutrophils were primed by preincubation with GM-CSF before the addition of synovial fluid, however, the kinetics of oxidant production were altered. Addition of GM-CSF to primed neutrophil suspensions activated a biphasic luminol chemiluminescence response characterised by an additional transient oxidase activity detected two to three minutes after stimulation (fig 1A). Similarly, priming of neutrophils before exposure to synovial fluid also altered oxidase activity as detected by the cytochrome c assay (fig 1B): by contrast with that found in unprimed cells, cell free synovial fluid activated a rapid, transient burst of $\mathrm{O}_{2}^{-}$secretion in primed cells. Thus cell free synovial fluid activates only intracellular oxidant production in unprimed neutrophils, but in primed cells an additional phase of reactive oxidant secretion is also seen.

We have previously shown that the transient phase of luminol chemiluminescence, only detectable on activation of primed neutrophils, is due to the presence of soluble immunoglobulin aggregates within synovial fluid. Thus it was necessary to determine if these soluble aggregates were responsible for the activation of oxidant secretion in primed cells. Centrifugation of synovial fluid at $11600 \mathrm{~g}$ for
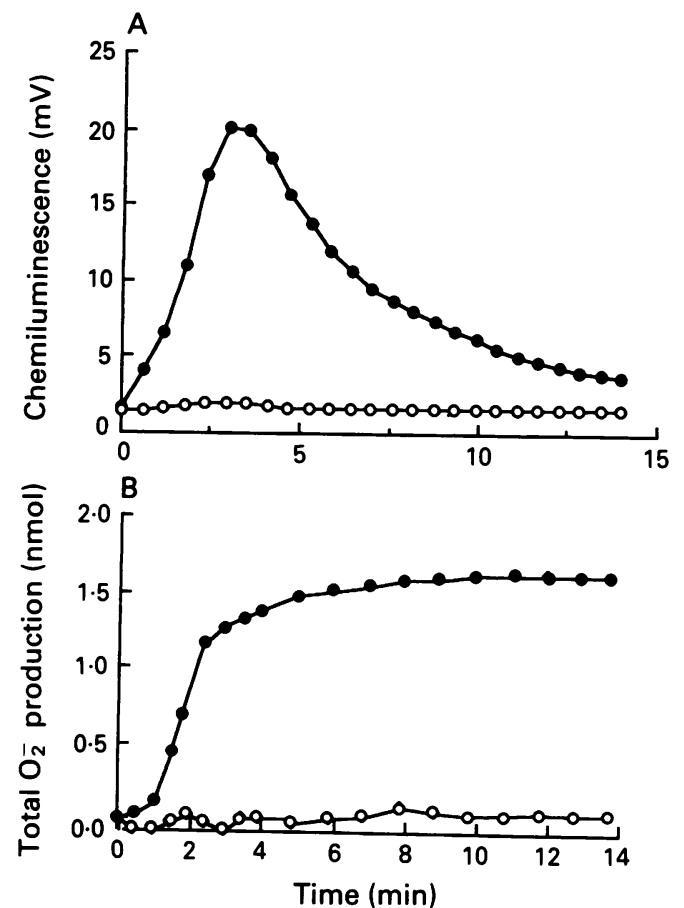

Figure 2 Activation of neutrophils by the soluble fraction of rheumatoid synovial fluid. Neutrophils $\left(10^{7} / \mathrm{ml}\right)$ were obtained and suspended as described in the legend to fig 1. After incubation for one hour at $37^{\circ} \mathrm{C}, 5 \times 10^{5}$ cells were activated with $10 \%(\mathrm{v} / \mathrm{v})$ final concentration of the supernatant of cell free synovial fluid, and the oxidants produced measured by $(A)$ chemiluminescence ( $L K B 1251$ luminometer) and (B) cytochrome c reduction at $550 \mathrm{~nm}$ (Bio-Rad 3550 kinetic plate reader). The supernatant was obtained by centrifugation of the cell free synovial fluid at $11600 \mathrm{~g}$ for two minutes. In (A) $10 \mu \mathrm{M}$ luminol was present and the volume was made up to $1 \mathrm{ml}$ with $R P M I$ 1640 medium. In (B) $75 \mu M$ cytochrome $c$ was present and the volume was made up to $200 \mu \mathrm{l}$ with RPMI 1640 medium: reference wells contained the same additions plus $30 \mu \mathrm{g} / \mathrm{ml}$ superoxide dismutase. Both assays were performed at $37^{\circ} \mathrm{C}$. Similar results were obtained in at least 20 further experiments with different synovial fluid samples and neutrophils from different donors.

two minutes produced pellets of the insoluble immunoglobulin aggregates and the only factors present within the supernatant that significantly activated reactive oxidant production were the soluble aggregates. ${ }^{15}$ Addition of the supernatant fraction did not activate either luminol chemiluminescence or $\mathrm{O}_{2}^{-}$secretion in unprimed neutrophils (fig 2). In cells that had previously been primed with GM-CSF, however, these soluble aggregates activated luminol chemiluminescence, but also activated the secretion of substantial quantities of $\mathrm{O}_{2}^{-}$. Indeed, the concentrations of $\mathrm{O}_{2}^{-}$ secreted by these soluble aggregates were comparable with the concentrations secreted by unfractionated synovial fluid. Under these experimental conditions, the resuspended pellet (containing the insoluble immunoglobulin aggregates) activated a monophasic luminol chemiluminescence response, but did not activate $\mathrm{O}_{2}^{-}$secretion in either primed or unprimed cells (fig 3 ). Increasing the concentration of insoluble immunoglobulin aggregates to $50 \%(\mathrm{v} / \mathrm{v})$, however, altered the kinetics of oxidant production (fig 4): the maximal rate of luminol chemiluminescence occurred more quickly after stimulation of cells that had previously been primed (fig 4A). Furthermore, this higher concentration of 

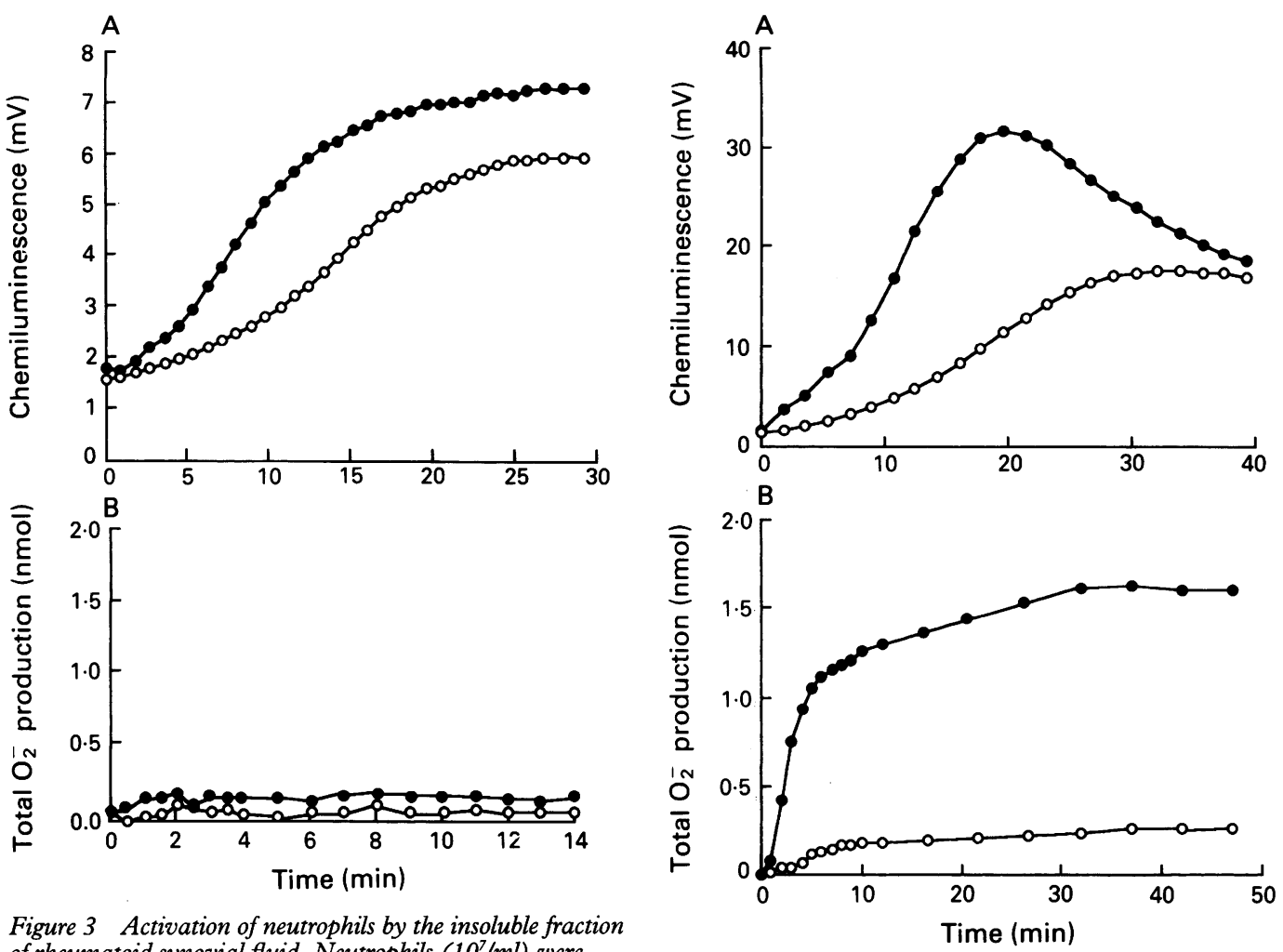

Figure 3 Activation of neutrophils by the insoluble fraction of rheumatoid synovial fluid. Neutrophils $\left(10^{7} / \mathrm{ml}\right)$ were obtained and suspended as described in the legend to fig 1 . After incubation for one hour at $37^{\circ} \mathrm{C}, 5 \times 10^{5}$ cells were activated with $10 \%(\mathrm{v} / \mathrm{v})$ final concentration of the pellet fraction of cell free synovial fluid, and the oxidants

produced were measured by $(A)$ chemiluminescence (LKB 1251 luminometer) and (B) cytochrome c reduction at 550 nm (Bio-Rad 3550 kinetic plate reader). The pellet was obtained by centrifugation of the cell free synovial fluid at $11600 \mathrm{~g}$ for two minutes, the supernatant was removed, and the pellet was resuspended in a volume of RPMI 1640 equivalent to that of the original fluid. In (A) $10 \mu \mathrm{M}$ luminol was present and the volume was made up to $1 \mathrm{ml}$ with RPMI 1640 medium. In (B) $75 \mu M$ cytochrome $c$ was present and the volume was made up of $200 \mu \mathrm{l}$ with RPMI 1640 medium; reference wells contained the same additions plus $30 \mu \mathrm{g} / \mathrm{ml}$ superoxide dismutase. Both types of assay were performed at $37^{\circ} \mathrm{C}$. Similar results were obtained in at least 20 further experiments with different synovial fluid samples and neutrophils from different donors.

insoluble aggregates activated low but detectable $\mathrm{O}_{2}^{-}$secretion in unprimed cells, but this secretion was enhanced if the cells were previously exposed to GM-CSF (fig 4B).

CONCENTRATION DEPENDENCE OF THE ABILITY OF SOLUBLE AND INSOLUBLE

IMMUNOGLOBULIN AGGREGATES TO ACTIVATE REACTIVE OXIDANT GENERATION

Because $50 \%$ but not $10 \%$ (v/v) of insoluble immunoglobulin aggregates activated $\mathrm{O}_{2}^{-}$ secretion in primed neutrophils, it was necessary to establish the effect of varying concentrations of both the soluble and insoluble aggregates on neutrophil activation. Hence, neutrophils were incubated in the presence and absence of GM-CSF and chemiluminescence and cytochrome $c$ reduction was measured in response to varying concentrations of these aggregates. When unprimed neutrophils were incubated with the soluble aggregates, no concentration tested $(0 \cdot 1 \%-100 \%, \mathrm{v} / \mathrm{v})$ activated luminol chemiluminescence or $\mathrm{O}_{2}^{-}$secretion (fig 5). If these cells were first primed with GM-CSF then the

Figure 4 Activation of neutrophils by the insoluble fraction of rheumatoid synovial fluid. Neutrophils were incubated as described in the legend to fig 3 except that stimulation was achieved by the addition of 50\% (final, v/v) of the resuspended pellet. Similar results were obtained in at least 20 further experiments with different synovial fluid samples and neutrophils from different donors.

soluble complexes activated maximal chemiluminescence and $\mathrm{O}_{2}^{-}$secretion at a concentration of $10 \%(\mathrm{v} / \mathrm{v})$. At concentrations above this, the rates of reactive oxidant production actually decreased, perhaps owing to down regulation of the response or else scavenging of oxidants by the multitude of proteins present within these crude supernatants.

When the insoluble immunoglobulin aggregates were tested on unprimed cells, maximal chemiluminescence was found at or above $30 \%(\mathrm{v} / \mathrm{v})$ and a low $\mathrm{O}_{2}^{-}$secretion was also found at these concentrations (fig 6). In primed cells, chemiluminescence was enhanced above $50 \%(\mathrm{v} / \mathrm{v})$ and although low concentrations did not stimulate much $\mathrm{O}_{2}^{-}$ secretion, concentrations of $25 \%$ and above stimulated substantial rates of cytochrome $c$ reduction.

STIMULATION OF ENZYME RELEASE BY IMMUNOGLOBULIN AGGREGATES

To determine if the immunoglobulin aggregates stimulated the release of granule enzymes from unprimed neutrophils, the secretion of myeloperoxidase was determined. After incubation with GM-CSF for 60 minutes at $37^{\circ} \mathrm{C}$, neutrophil suspensions were pretreated with cytochalasin B $(5 \mu \mathrm{g} / \mathrm{ml})$ and then stimulated (at time zero) with $1 \mu \mathrm{M}$ fMetLeu-Phe, or $10 \%$ soluble or insoluble immunoglobulin aggregates. The release of myeloperoxidase into the extracellular medium 

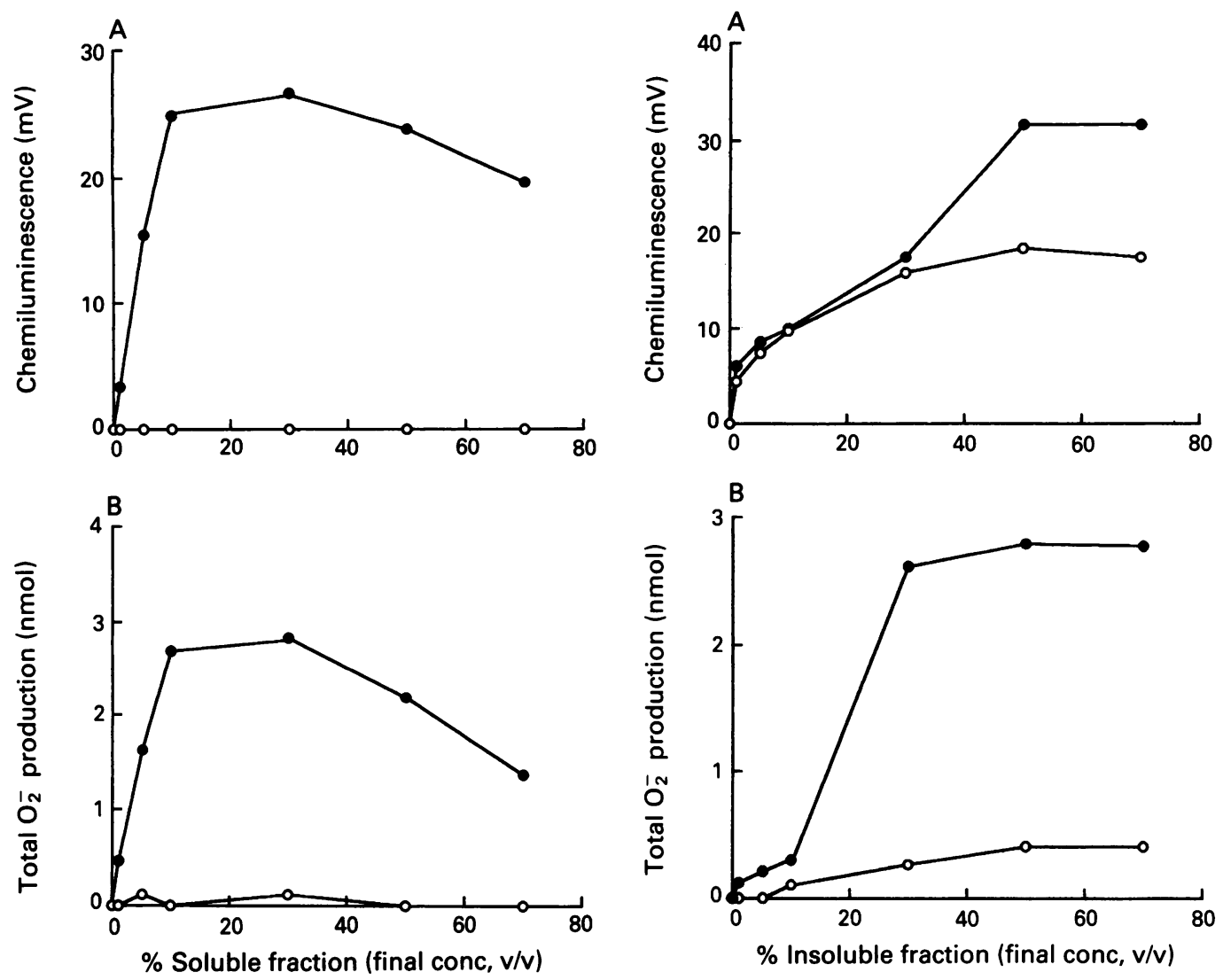

Figure 5 Activation of neutrophil reactive oxidant production by the addition of varying concentrations of the soluble fraction of cell free synovial fluid. Neutrophils were incubated as described in the legend to fig 2 except that stimulation was achieved by the addition of varying volumes of supernatants obtained after centrifugation of cell free synovial fluids. Similar results were obtained in two further experiments with different synovial fluid samples and neutrophils from different donors.

was measured at intervals. fMet-Leu-Phe stimulated a rapid (within 30 seconds) release of this enzyme into the medium. This accounted for $25-35 \%$ of the total cellular activity (fig 7). Whereas the soluble immunoglobulin aggregates also stimulated the release of this enzyme with similar kinetics, the insoluble complexes did not cause degranulation. Increasing the concentration of these insoluble complexes to $25 \%$ (v/v) did not lead to detectable enzyme secretion. In the absence of cytochalasin B, both fMet-Leu-Phe and the soluble aggregates stimulated low, but detectable secretion $(0 \cdot 15(\mathrm{SD} 0.05) \mathrm{mU}, \mathrm{n}=3$, and $0.08(0.03) \mathrm{mU}$ respectively). By contrast, the insoluble aggregates at concentrations of $0 \cdot 1-50 \%(\mathrm{v} / \mathrm{v})$ did not stimulate myeloperoxidase release. In the absence of GM-CSF and cytochalasin B all agonists used stimulated insignificant amounts of myeloperoxidase secretion.

\section{Discussion}

We have previously shown that the two major neutrophil activating factors within synovial fluid of patients with rheumatoid arthritis are attributable to soluble and insoluble immunoglobulin aggregates. ${ }^{15}$ This conclusion has been reached with protein A affinity chromatography, which isolates the insoluble

Figure 6 Activation of neutrophil reactive oxidant production by the addition of varying concentrations of the insoluble fraction of cell free synovial fluid. Neutrophils were incubated exactly as described in the legend to fig 3 except that stimulation was achieved by the addition of varying concentrations of the resuspended insoluble fraction obtained after centrifugation of cell free synovial fluid. Similar results were obtained in two further experiments with different synovial fluid samples and neutrophils from differen donors.

aggregates (which are almost completely recovered in the pellet after centrifugation at $11600 \mathrm{~g}$ for two minutes) and also the soluble

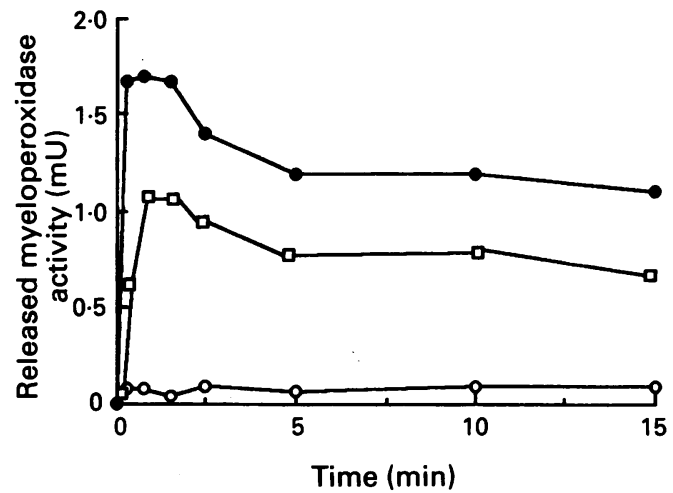

Figure 7 Myeloperoxidase release after neutrophil activation. Suspensions of neutrophils in RPMI 1640 medium $\left(10^{7} / \mathrm{ml}\right)$ were primed with $50 \mathrm{U} / \mathrm{ml}$ granulocytemacrophage colony stimulating factor (GM-CSF) for one hour at $37^{\circ} \mathrm{C}$. After this incubation, $5 \mu \mathrm{g} / \mathrm{ml}$ cytochalasin $B$ was added to the suspensions before stimulation with $1 \mu M$ fMet-Leu-Phe ( $), 10 \%(v / v)$ of the supernatant from cell free synovial fluid $(\square)$, or $10 \%(v / v)$ the pellet from cell free synovial fluid $(0)$. The pellet and supernatant were obtained by centrifugation of cell free synovial fluid for two minutes at $11600 \mathrm{~g}$. After stimulation, $5 \times 10^{5}$ cells were sampled at varying time points, the cells rapidly spun down, and the extracellular medium assayed for myeloperoxidase activity. Similar results were obtained in three other experiments with different synovial fluid samples and neutrophils from different donors. 
aggregates that are present in the supernatant after centrifugation. It is interesting to note that although synovial fluid has been reported to contain various factors capable of activating neutrophils (such as complement fragments, leukotrienes, interleukin 8) protein A affinity chromatography removes all factors capable of activating reactive oxidant production by neutrophils in our assays. Hence, the potential role of these neutrophil activating factors, other than the immunoglobulin aggregates or immune complexes in the pathology of joint disease is questionable. Such factors, although they may not activate neutrophils themselves, may alter the responsiveness of these cells to the immunoglobulin aggregates. Alternatively, such factors may bind to the immunoglobulin aggregates or the protein A column and hence be non-specifically removed from synovial fluid by this procedure.

Because the soluble and insoluble immunoglobulin aggregates seemed to activate neutrophils through distinct mechanisms, the aim of this study was to determine if either of these complexes activated the secretion of reactive oxidants and granule enzymes. It is now established that neutrophils can generate reactive oxidants both intracellularly and extracellularly. ${ }^{23} 24$ Intracellular oxidant production (which accompanies phagocytosis of large particles such as bacteria or large insoluble immune complexes) is obviously required for the killing of ingested pathogens. On the other hand, activation of the NADPH oxidase on the plasma membrane, which usually accompanies activation by soluble agonists, ${ }^{25}$ will lead to oxidant secretion and this is likely to result in damage to host tissues. Such damage will be exacerbated if secretion of oxidants occurs in parallel with the secretion of cytotoxic enzymes via degranulation. Hence, if products derived from neutrophils contribute to the joint damage associated with rheumatoid arthritis, then it is likely to be the factors in synovial fluid that activate secretion which are important in disease.

Both the soluble and insoluble immunoglobulin aggregates activated the secretion of reactive oxidants from blood neutrophils, but the soluble factors were potent at concentrations at least 10 -fold lower than were required for the insoluble complexes. What is also remarkable is that the neutrophils need to be primed before the secretion of substantial amounts of reactive oxidants can occur. Indeed, when the neutrophils are primed, the extracellular secretion of myeloperoxidase can occur (albeit at low but significant rates in the absence of cytochalasin B) on stimulation by the soluble, but not the insoluble immunoglobulin aggregates. These findings are of obvious importance because it is generally assumed that for secretion to occur in activated neutrophils, they must first be incubated with cytochalasins that disrupt assembly of microfilaments. Hence, the finding that exposure to cytokines such as GM-CSF followed by activation with soluble immunoglobulin aggregates results in secretion of oxidants and granule enzymes is of profound importance in understanding the molecular events that occur within inflamed joints. Synovial fluid contains cytokines to prime the neutrophils ${ }^{26} 27$ plus soluble immunoglobulin aggregates that activate a rapid and transient release of tissue damaging reactive oxidants and granule enzymes. We thus propose that these processes are of great importance in disease.

While this work indicates a mechanism whereby newly recruited neutrophils will secrete tissue damaging products, it also raises important, but as yet unexplained issues. Firstly, the size of an immune complex or immunoglobulin aggregate possibly determines the mechanism by which it activates neutrophils. Large complexes seem to be primarily phagocytosed, especially at low concentrations: at high concentrations secretion may occur because of 'frustrated phagocytosis' or cell lysis. On the other hand, soluble complexes seem to primarily activate secretion in primed cells. Secondly, for secretion to occur the cells must be primed. Priming does not appreciably affect the level of expression of Fc $\gamma$ RII or Fc $\gamma$ RIII, the plasma membrane receptors ${ }^{28}$ that interact with these immunoglobulin aggregates: perhaps priming alters the affinity (rather than number) of receptors on the plasma membrane, alters their distribution (for example, induces clustering or allows Fc $\gamma$ RII and III to interact) or affects the mechanisms by which these receptors are coupled to their intracellular signal transduction systems. Further work is necessary to investigate these possibilities. Thirdly, the kinetics of activation, the proportions of intra and extracellular oxidase activity, and the sensitivity of activation to staurosporine $e^{15}$ indicate distinct activation mechanisms regulating the response of neutrophil to the soluble and insoluble immunoglobulin aggregates. These findings raise the intriguing possibility that the receptors or signal transduction systems activated by these two types of immunoglobulin aggregates are different. This suggests that it may be feasible to block activation of neutrophils via the soluble immunoglobulin aggregates, but leave opsonophagocytosis unaffected. Further work is necessary to establish this possibility, which if realised, should enable us to prevent secretion within the rheumatoid joint (which will prevent tissue damage) but not compromise the ability of these patients to combat bacterial infections.

We thank the Arthritis and Rheumatism Council and Mersey Regional Health Authority for financial support.

\footnotetext{
1 Halliwell B, Gutteridge J M C. Free radicals in biology and medicine. Oxford: Clarendon Press, 1985.

2 mewn K A. The polymorphonuclear cell in rheumatoid arthritis. Br $\mathcal{f}$ Rheumatol 1988; 27: 150-5.

3 Nurcombe H L, Bucknall R C, Edwards S W. Neutrophils isolated from the synovial fluid of patients with rheumatoid arthritis: priming and activation in vivo. Ann Rheum Dis 1991; 50: 147-53.

4 Emery P, Lopez A F, Burns G F, Vadas M A. Synovial fluid neutrophils of patients with rheumatoid arthritis have membrane antigen changes that reflect activation. $A n n$ Rheum Dis 1988; 47: 34-9.

5 Breedveld F C, Lafeber G J M, van den Barselaar T T, van Dissel J T, Leijh P C J. Phagocytosis and intracellula killing of Staphylococcus aureus by polymorphonuclea cells from synovial fluid of patients with rheumatoid arthritis. Arthritis Rheum 1986; 29: 166-73.
} 
6 Dularay B, Elson C J, Dieppe P A. Enhanced oxidative response of polymorphonuclear leukocytes from synovial fluids of patients with rheumatoid arthritis. Autoimmunity 1988; 1: 159-69.

7 Merry P, Winyard P G, Morris C J, Grootveld M, Blake D R. Oxygen free radicals, inflammation and synovitis: the current status. Ann Rheum Dis 1989; 48: 864-70.

8 Stevens C R, Williams R B, Farrell A J, Blake D R. Hypoxia and inflammatory synovitis: observations and speculation. Ann Rheum Dis 1991; 50: 124-32.

9 Edwards S W, Hughes V, Barrow J, Bucknall R. Immunological detection of myeloperoxidase in synovia fluid from patients with rheumatoid arthritis. Biochem $f$ 1988; 250: 81-5.

10 Nurcombe H L, Bucknall R C, Edwards S W. Activation of the myelperoxidase- $\mathrm{H}_{2} \mathrm{O}_{2}$ system by synovial fluid isolated from patients with rheumatoid arthritis. Ann Rheum Dis 1991; 50: 237-42.

11 Dularay B, Badesha J S, Dieppe P A, Elson C J. Oxidative response of polymorphonuclear leucocytes to synovial fluids from patients with rheumatoid arthritis. Ann Rheum Dis 1990; 49: 661-4

12 Gale R, Bertouch J V, Bradley J, Roberts-Thomsen P J. Direct activation of neutrophil chemiluminescence by rheumatoid sera and synovial fluid. Ann Rheum Dis 1983; 42: $158-62$.

13 Blackburn W D Jr, Koopman W J, Schrohenloher R E, Heck L. Induction of neutrophil enzyme release by rheumatoid factors: evidence for differences based on molecular factors: evidence for differences based on molecular characterist

14 Robinson J J, Watson F, Bucknall R C, Edwards S W. Stimulation of neutrophils by insoluble immunoglobulin aggregates from synovial fluid of patients with rheumatoid arthritis. Eur $\mathcal{Y}$ Clin Invest 1992; 22: 314-8.

15 Robinson J J, Watson F, Bucknall R C, Edwards S W. Activation of neutrophil reactive-oxidant production by synovial fluid from patients with inflammatory joint synove Soluble and insoluble immunoglobulin aggregates activate different pathways in primed and aggregates activate different pathways in p
unprimed cells. Biochem $\mathcal{f} 1992 ; 286: 345-51$.

16 Edwards S W, Say J E, Hart C A. Oxygen-dependent killing of Staphlococcus aureus by human neutrophils. $\mathcal{F}$ Gen Microbiol 1987; 133: 3591-97.
17 Edwards S W, Holden C S, Humphreys J M, Hart C A. Granulocyte-macrophage colony-stimulating factor (GM-CSF) primes the respiratory burst and stimulates protein biosynthesis in human neutrophils. FEBS Lett 1989; 256: 62-6.

18 Edwards S W. Luminol- and lucigenin-dependent chemiluminescence of neutrophils: role of degranulation. chemiluminescence of neutrophils:

19 Babior B M, Kipnes R S, Curnutte J T. Biological defense mechanisms: the production by leukocytes of superoxide, a potential bacteriocidal agent. $\mathcal{F}$ Clin Invest 1973; 52: 741-7.

20 Edwards S W, Nurcombe N L, Hart C A. Oxidative inactivation of myeloperoxidase released from human neutrophils. Biochem f 1987; 245: 925-8.

21 Putte J. Peroxidases. In: Bergmeyer H U, ed. Methods of enzymatic analysis. Weinheim: Verlag-Chemie, 1974: enzymatic

22 Dahlgren C, Aniansson H, Magnusson K-E. Pattern of formylmethionyl-leucyl-phenylalanine-induced luminoland lucigenin-dependent chemiluminescence in human neutrophils. Infect Immun 1985; 47: 326-8.

23 Nurcombe H L, Edwards S W. Role of myeloperoxidase in intra- and extra-cellular chemiluminescence of neutrophils. Ann Rheum Dis 1989; 48: 56-62.

24 Briheim G, Stendahl O C D. Intra- and extracellular events in luminol-dependent chemiluminescence of polymorphonuclear leukocytes. Infect Immun 1984; 45: 1-5.

25 Watson F, Robinson J J, Edwards S W. Protein kinase C dependent and independent activation of the NADPH oxidase of human neutrophils. $\mathcal{F}$ Biol Chem 1991; 266: 7432-9.

26 Feldmann M, Brennan F M, Chantry D, et al. Cytokine production in the rheumatoid joint: implications for treatment. Ann Rheum Dis 1990; 49: 480-6.

27 Westacott C I, Whicher J T, Barnes I C, Thompson D, Swan A J, Dieppe P A. Synovial fluid concentration of five different cytokines in rheumatic diseases. Ann Rheum Dis 1990; 49: 676-81.

28 Edwards S W, Watson F, MacLeod R, Davies J. Receptor expression and oxidase activity in human neutrophils: regulation by granulocyte-macrophage colonystimulating factor and dependence upon protein biosynthesis. Biosci Rep 1990; 10: 393-401. 